\title{
Clinical Characteristics of the Causes of Plantar Heel Pain
}

\author{
Tae Im Yi, M.D., Ga Eun Lee, M.D., In Seok Seo, M.D., Won Seok Huh, M.D., \\ Tae Hee Yoon, M.D., Bo Ra Kim, M.D.
}

Department of Rehabilitation Medicine, Bundang Jesaeng General Hospital, Seongnam 463-774, Korea

\begin{abstract}
Objective The objectives of this study were to investigate the causes of plantar heel pain and find differences in the clinical features of plantar fasciitis (PF) and fat pad atrophy (FPA), which are common causes of plantar heel pain, for use in differential diagnosis.

Method This retrospective study analyzed the medical records of 250 patients with plantar heel pain at the Foot Clinic of Rehabilitation Medicine at Bundang Jesaeng General Hospital from January to September, 2008.

Results The subjects used in this study were 114 men and 136 women patients with a mean age of 43.8 years and mean heel pain duration of 13.3 months. Causes of plantar heel pain were PF (53.2\%), FPA (14.8\%), pes cavus (10.4\%), PF with FPA (9.2\%), pes planus (4.8\%), plantar fibromatosis (4.4\%), plantar fascia rupture (1.6\%), neuropathy $(0.8 \%)$, and small shoe syndrome $(0.8 \%)$. PF and FPA were most frequently diagnosed. First-step pain in the morning, and tenderness on medial calcaneal tuberosity correlated with PF. FPA mainly involved bilateral pain, pain at night, and pain that was aggravated by standing. Heel cord tightness was the most common biomechanical abnormality of the foot. Heel spur was frequently seen in X-rays of patients with PF.

Conclusion Plantar heel pain can be provoked by PF, FPA, and other causes. Patients with PF or FPA typically show different characteristics in clinical features. Plantar heel pain requires differential diagnosis for appropriate treatment.
\end{abstract}

Key Words Plantar heel pain, Plantar fasciitis, Fat pad atrophy

Received April 27, 2010; Accepted February 23, 2011

Corresponding author: Ga Eun Lee

Department of Rehabilitation Medicine, Dajin Medical Center Bundang Jesaeng General Hospital, 255-2, Seohyeon-dong, Bundang-gu, Seongnam 463-774, Korea

Tel: +82-31-779-0395, Fax: +82-31-779-0635, E-mail: smart0416@hotmail. com

(c) This is an open-access article distributed under the terms of the Creative Commons Attribution Non-Commercial License (http://creativecommons.org/ licenses/by-nc/3.0) which permits unrestricted noncommercial use, distribution, and reproduction in any medium, provided the original work is properly cited. Copyright $\odot 2011$ by Korean Academy of Rehabilitation Medicine

\section{INTRODUCTION}

Plantar heel pain in adulthood is the most common problem of the foot, and can be provoked by bone, soft tissue, nerve, or systemic disease. ${ }^{1,2}$ Although accurate diagnosis and appropriate management are important, distinguishing the various causes of highly similar symptoms is difficult. Plantar heel pains from skeletal problems are caused by calcaneal stress fracture, apophysitis of the calcaneus (Sever's disease), osteomyelitis, or inflammatory arthropathy. Soft tissue pathology includes fat pad atrophy (FPA) or contusion, plantar fascia rupture and plantar fasciitis (PF). Heel pain may be induced 
by neural causes such as entrapment or compression of the first branch of the lateral plantar nerve (Baxter's nerve), medial calcaneal branch of posterior tibial nerve, or nerve to abductor digiti quinti muscle. Other neural causes include S1 radiculopathy, tarsal tunnel syndrome, and peripheral neuropathy. ${ }^{1,3}$

$\mathrm{PF}$ is reported to be the most common cause of plantar heel pain. ${ }^{1}$ The pathology is still unknown, but histopathologic examination of biopsy specimens from patients undergoing excessive extension or microinjuries of the plantar fascia shows secondary degenerative changes in the plantar fascia, with or without fibroblastic proliferation, and without acute inflammation. ${ }^{4}$ The peak age of incidence in the general population is between 40 and 60 years. The risk factors for PF include running excessively (or suddenly increasing running distance), occupations that involve long periods of standing, pes planus, limited ankle dorsiflexion, and obesity. ${ }^{1,3,5}$ FPA occurs after 40 years because of atrophy and thinning of the fat pad in the inferior heel, with loss of water, collagen, and elastic tissue, reducing shock absorbency and protection of the calcaneus. $^{3}$

Differential diagnosis between PF and FPA is important for proper treatment. Baxter and Pfeffer ${ }^{6}$ determined differential diagnosis according to different tender sites that were closely related to the origins of heel pains. Therefore, we studied the variable causes of plantar heel pain and clinical characteristics of PF and FPA. We generated basic data for differential diagnosis using detailed history taking and thorough physical examination.

\section{MATERIALS AND METHODS}

\section{Subjects}

A retrospective study of the medical records of 250 patients with plantar heel pain was conducted at the Foot Clinic of Rehabilitation Medicine at Bundang Jesaeng General Hospital from January to September, 2008. We excluded 25 patients due to insufficient medical records, physical examination, or sonographic findings. Others were excluded for a history of musculoskeletal disease that caused heel pain, neurologic pathologies such as S1 radiculopathy, or systemic disease such as inflammatory or rheumatic disease. We included 250 patients whose medical records were sufficient to be used in the study.

\section{Study procedures}

To standardize symptoms of plantar heel pains, the patients filled out a questionnaire about their symptoms. We reviewed their medical records and results from physical examinations, glass plate with a reflecting mirror, static and dynamic plantar pressure measurement, $\mathrm{X}$-ray and ultrasonography. Patients were registered if two or fewer criteria were absent, and which were treated as missing values.

We investigated age, gender, and diagnoses of patients with heel pain and compared patients with PF and FPA for pain characteristics, and the results of physical examinations and X-rays. An experienced physiatrist confirmed PF or FPA using musculoskeletal sonographic findings.

We diagnosed PF when the patient had tenderness on a medial calcaneal tuberosity and a hypoechoic fusiformshaped swelling more than $4 \mathrm{~mm}$ in thickness at the origin of the plantar fascia. ${ }^{7}$ We diagnosed FPA when the patient had a fat pad that was less than $3 \mathrm{~mm}$ in thickness by ultrasonographic evaluation and met more than three of the following criteria: pain at heel center or margin, worsening pain when barefoot or after a long period of standing, or tenderness on the heel center or palpable calcaneus. We chose $3 \mathrm{~mm}$ as the standard fat pad thickness because it was less than 2 standard deviations from the normal value of the mean, according to the results of a sonographic study on normal adults. ${ }^{8}$

We investigated the characteristics, timing and duration of the pain, relieving or aggravating factors, and bilaterality, to determine the features of PF and FPA. Pes planus, pes cavus and heel cord tightness were determined by physical examination, and heel spurs were checked by $\mathrm{X}$-ray. The absolute value of a talometatarsal angle over 4 degrees was considered abnormal. ${ }^{9}$ Pes planus was diagnosed when the talometatarsal angle was below -4 degrees; medial longitudinal arch was absent or low; the medial side of the foot was straightened or low on visual inspection; or weight-bearing caused a depressed midfoot, supinated foot or calcaneal valgus, and medially displaced the weight-bearing axis to the mid-axis. Pes cavus was diagnosed when the talometatarsal was over 4 degrees, calcaneal varus was observed, and high pressure occurred underneath the forefoot and heel. Heel cord tightness was defined as a limited ankle dorsiflexion below 10 degrees. ${ }^{5}$ 
Table 1. Characteristics of Subjects $(\mathrm{N}=250)$

\begin{tabular}{lccc}
\multicolumn{1}{c}{ Characteristics } & Male & Female & Total \\
\hline Number of patients & 114 & 136 & 250 \\
Age (years) & $39.1 \pm 11.7$ & $47.7 \pm 10.9^{*}$ & $43.8 \pm 12.0$ \\
Duration of symptom (months) & $13.5 \pm 19.5$ & $13.1 \pm 15.5$ & $13.3 \pm 17.4$
\end{tabular}

Values are mean \pm standard deviation

${ }^{*} \mathrm{p}<0.05$

\section{Data analysis}

SPSS 12.0 for Windows was used for statistical analysis. Odds ratios by multinomial logistic regression were used to predict specific diagnosis using clinical characteristics, physical examination, or radiologic findings of $\mathrm{PF}$ or FPA patients. To define the features of patients with both PF and FPA (PFFPA), we analyzed odds ratios between patients with PF and with PFFPA, and between patients with FPA and with PFFPA.

\section{RESULTS}

\section{Characteristics of subjects}

The mean age of 114 men and 136 women was 43.8 years, and women were older than men on average. The mean duration of pain was 13.3 months (Table 1 ).

\section{Frequency of diagnosis}

PF was the most common diagnosis (113 subjects, $53.2 \%$ ). The second most common was FPA (37 subjects, $14.8 \%)$. PFFPA occurred in 26 cases (9.2\%), plantar fibromatosis in $11(4.4 \%)$, plantar fascia rupture in $4(1.6 \%)$ and neuropathy in $2(0.8 \%)$. Plantar heel pains were caused by pes cavus in 26 cases $(10.4 \%)$ and pes planus in 26 (4.8\%) with normal findings by ultrasonography and electrodiagnostic study, and without evidences of other pathologies (Table 2).

\section{Pain characteristics}

Patients with PF commonly experienced first-step pain in the morning (88.0\%) and relief of pain after walking (45.9\%). Unilateral pain was more common than bilateral (76.7\%), and $51.5 \%$ of patients with PF had pain duration of less than 6 months.

Patients with FPA suffered from not only aching pain (78.4\%) but also tingling (8.1\%), cold (5.4\%) and burning sensations $(1.8 \%)$. They complained of pain after a long walk (62.2\%), pain at night (13.5\%) and resting pain
Table 2. Diagnosis of Patients with Plantar Heel Pain

\begin{tabular}{lc}
\hline \multicolumn{1}{c}{ Diagnosis } & Number of patients (\%) \\
\hline PF & $133(53.2 \%)$ \\
FPA & $37(14.8 \%)$ \\
Pes cavus & $26(10.4 \%)$ \\
PFFPA & $23(9.2 \%)$ \\
Pes planus & $12(4.8 \%)$ \\
Plantar fibromatosis & $11(4.4 \%)$ \\
Plantar fascia rupture & $4(1.6 \%)$ \\
Neuropathy & $2(0.8 \%)$ \\
Small shoe syndrome & $2(0.8 \%)$ \\
\hline
\end{tabular}

PF: Plantar fasciitis, FPA: Fat pad atrophy, PFFPA: PF with FPA

(13.5\%). Bilateral pain was more common than unilateral pain $(78.4 \%)$. In $61.1 \%$ of patients with FPA, pain duration was over 6 months, and $22 \%$ of these patients had pain duration of over 2 years (Table 3 ).

\section{Abnormal foot biomechanics}

Heel cord tightness was the most common biomechanical problem and limited ankle dorsiflexion was more frequently observed in patients with PF (53.4\%) than in patients with FPA (35.1\%) ( $\mathrm{p}=0.049)$. The number of patients with pes planus was greater than that of patients with pes cavus in each group (PF $30.1 \%$, FPA 32.4\%, PFFPA 34.8\%) (Table 4).

\section{Heel spur by X-ray}

We detected heel spurs in PF patients using X-ray (38\%) (Table 4). Heel spur occurred more frequently in PF patients than in FPA patients according to a student $t$-test $(\mathrm{p}=0.001)$.

\section{Risk factors for PF or FPA}

PF and FPA were compared by multinomial logistic regression. Patients with FPA were likely to have pain 
Tae Im Yi, et al.

Table 3. Characteristics of Plantar Heel Pain

\begin{tabular}{|c|c|c|c|}
\hline \multirow{2}{*}{ Characteristics } & \multicolumn{3}{|c|}{ Number of patients (\%) } \\
\hline & PF & FPA & PFFPA \\
\hline \multicolumn{4}{|l|}{ Nature of pain } \\
\hline Tingling & $3(2.3 \%)$ & $3(8.1 \%)$ & $1(4.3 \%)$ \\
\hline Cold & - & $2(5.4 \%)$ & - \\
\hline Burning & $5(3.8 \%)$ & $7(1.8 \%)$ & $2(8.7 \%)$ \\
\hline Aching & $130(97.7 \%)$ & $29(78.4 \%)$ & $20(87.0 \%)$ \\
\hline \multicolumn{4}{|l|}{ Timing of pain } \\
\hline $1^{\text {st }}$ morning step pain & $117(88.0 \%)$ & $16(43.2 \%)$ & $17(73.9 \%)$ \\
\hline Pain relief by walking & $61(45.9 \%)$ & $7(18.9 \%)$ & $7(30.4 \%)$ \\
\hline Pain after a long walk & $48(36.1 \%)$ & $23(62.2 \%)$ & $11(47.8 \%)$ \\
\hline More pain during standing & $3(2.3 \%)$ & $11(29.7 \%)$ & $3(13.0 \%)$ \\
\hline Night pain & $4(3.0 \%)$ & $5(13.5 \%)$ & - \\
\hline Resting pain & $3(2.3 \%)$ & $5(13.5 \%)$ & - \\
\hline \multicolumn{4}{|l|}{ Bilaterality of pain } \\
\hline Unilateral pain & $102(76.7 \%)$ & $8(21.6 \%)$ & $14(60.9 \%)$ \\
\hline Bilateral pain & $31(23.3 \%)$ & $29(78.4 \%)$ & $9(39.1 \%)$ \\
\hline \multicolumn{4}{|l|}{ Duration of symptom } \\
\hline$\leq 6$ months & $67(51.5 \%)$ & $14(38.9 \%)$ & $13(59.1 \%)$ \\
\hline$>6$ and $\leq 24$ months & $55(42.3 \%)$ & $14(38.9 \%)$ & $4(18.2 \%)$ \\
\hline$>24$ months & $8(6.2 \%)$ & $8(22.2 \%)$ & $5(22.7 \%)$ \\
\hline
\end{tabular}

PF: Plantar fasciitis, FPA: Fat pad atrophy, PFFPA: PF with FPA

Table 4. Physical Examination and Heel Spur using X-ray

\begin{tabular}{|c|c|c|c|}
\hline \multirow{2}{*}{ Characteristics } & \multicolumn{3}{|c|}{ Number of patients (\%) } \\
\hline & PF & FPA & PFFPA \\
\hline \multicolumn{4}{|l|}{ Foot biomechanics } \\
\hline Pes planus & $40(30.1 \%)$ & $12(32.4 \%)$ & $8(34.8 \%)$ \\
\hline Pes cavus & $32(24.1 \%)$ & $7(18.9 \%)$ & $6(26.1 \%)$ \\
\hline Heel cord tightness & $71(53.4 \%)^{*}$ & $13(35.1 \%)$ & $13(56.5 \%)$ \\
\hline Heel spur in X-ray & $51(38.3 \%)^{*}$ & $5(13.5 \%)$ & $9(39.1 \%)$ \\
\hline
\end{tabular}

aggravated by prolonged standing (odds ratio=20.91), night pain (odds ratio=20.94) and bilateral pain (odds ratio $=24.95$ ) without first-step pain in the morning (odds ratio $=0.07)$ and tenderness on the medial calcaneal tuberosity (odds ratio=0.04). If the pain was bilateral and more severe with prolonged standing, it was likely to be caused by FPA. PFFPA was compared with PF and FPA by multinomial logistic regression. Morning first-step pain or medial calcaneal tenderness correlated with more sig-
Table 5. Risk Factors of Plantar Fasciitis or Fat Pad Atrophy

\begin{tabular}{lccc}
\hline & \multicolumn{3}{c}{ Odds ratio } \\
& (95\% Confidence interval) \\
\cline { 2 - 4 } & $\begin{array}{c}\text { PF vs } \\
\text { FPA }\end{array}$ & $\begin{array}{c}\text { PF vs } \\
\text { PFFPA }\end{array}$ & FPA vs \\
PFFPA \\
\hline $1^{\text {st }}$ morning step pain & 0.07 & 0.29 & 3.91 \\
More pain during standing & 20.91 & 6.23 & 0.30 \\
Night pain & 20.94 & - & - \\
Tenderness of medial heel & 0.04 & 0.21 & 5.07 \\
Bilateral pain & 24.95 & 2.69 & 0.11 \\
\hline PF: Plantar fasc
\end{tabular}

PF: Plantar fasciitis, FPA: Fat pad atrophy, PFFPA: PF with FPA

nificantly PFFPA than FPA (Table 5).

\section{DISCUSSION}

PF is reported to be the most common cause of pain in the inferior heel. It is estimated to account for $11-15 \%$ of all foot symptoms that require professional care among 
adults, ${ }^{10,11}$ and occurs in up to $10 \%$ of the US population. ${ }^{12}$ The incidence peaks between the age of 40 and 60 years in the general population. ${ }^{1,3} \mathrm{PF}$ was the most common diagnosis in this study, occurring in $53.2 \%$ of patients with plantar heel pain. PF is caused by repetitive pronatory stress that increases tensile force on the plantar fascia, causing the plantar arch to lower. The pronatory effect increases with age, and is related to limited ankle dorsiflexion caused by decreased elasticity of the tendons, and the reduced range of motion that occurs with age. PF usually has diverse causes. We investigated variable causes of plantar heel pain and their incidence. Interestingly, the fourth most common was PFFPA. Aging caused physiological changes leading to $\mathrm{PF}$, increasing stress on the calcaneus and plantar fascia because of loss of buffering tissue such as water and collagen of the fat pad. The fat pad atrophied and shock absorbency diminished in subjects that were older than 40 years. PF can be co-morbid with FPA, and this should be considered in designing treatment strategy. Our study had 38 people $(15.2 \%)$ with pes cavus or pes planus and no structural abnormality of the heel. They had been misdiagnosed as having PF and treated with conservative therapy including local steroid injection. Because patients with pes cavus or pes planus might develop sufficient plantar pain to require professional care at a hospital, either condition should be considered in differential diagnosis. Our study had four patients with plantar fascia rupture (1.6\%). One had heel pain after running on a treadmill, and the other three patients reported a past medical history of local steroid injection for heel pain. One of these three had two steroid injections and another developed pain during basketball game, 4 months after a steroid local injection. The other patient described an audible "pop" while walking 2 months after a local steroid injection.

The peak age of incidence of PF in the general population is between 40 and 60 years. ${ }^{1,3}$ In this study, the mean age of patients with PF was 44.7 years. PF patients under 40 years (42.9\%) were obese, or had occupations and leisure activities that could lead to heel pain. ${ }^{1,3,5}$ Younger patients may also be more interested in quality of life and heel pain.

Baxter and Pfeffer ${ }^{6}$ reported on 69 heels from 53 patients with chronic heel pain who had a surgical release of the first branch of the lateral plantar nerve. Postoperatively, 61 heels (89\%) had good or excellent results. Entrapment of the first branch of the lateral plantar nerve (Baxter's nerve) was not determined in this study, but 12 patients (15.3\%) with FPA and 3 (13.0\%) with PFFPA had tingling, cold, or burning sensations. Tingling or burning symptoms may be the result of neural causes. Nerves are localized in superficial regions where they can be easily compressed after atrophy of the heel fat pad. This makes them vulnerable to contusion if shock absorbency is reduced. Entrapment of the Baxter nerve was difficult to be examined objectively, so this study did not confirm that diagnosis, although this may have occurred among the subjects.

Because PF and FPA may share very similar symptoms and are difficult to distinguish from one another, we performed multinomial logistic regression for statistical modeling and predicting specific diagnoses. When we compared PF with FPA, first-step pain in the morning and tenderness on the medial calcaneal tuberosity tended to indicate $\mathrm{PF}$, and worsening pain during prolonged standing, pain at night, or bilateral pain were more likely to indicate FPA. However, $43.2 \%$ of FPA patients experienced first-step pain in the morning. Consequently, we should not make a diagnosis based on only one symptom, and should consider other symptoms, results of physical examinations, and radiologic evaluation. In case of mixed characteristics of PF and FPA (Table 5), PFFPA should be considered first and ultrasonography possibly used as an adjuvant diagnostic tool. Bilateral pain of PF patients was reported in $33 \%$ of patients with heel painl, and this occurred in $23.3 \%$ of patients in this study, but unilateral pain was still dominant. Bilateral pain was seen in $78.4 \%$ of patients with FPA, and was significantly more likely to indicate FPA than PF (odds ratio=57.22).

Limited ankle dorsiflexion on the involved side significantly increased the risk of $\mathrm{PF}^{5,13}$ Approximately 10 degrees of ankle dorsiflexion with the knee extended was required during a normal gait. ${ }^{14}$ If the Achilles tendon is shortened, limiting ankle dorsiflexion, excessive pronation of the foot may occur to compensate for this limitation. Excessive pronation of the foot increases tensile loads on the plantar aponeurosis. Because heel cord tightness of patients with PF was more frequently seen in patients with PF than in patients with FPA ( $p=0.049)$, the greater the limitation in ankle dorsiflexion, the more load on the plantar fascia. This provides a good target for treatment. Digiovanni et al. ${ }^{15}$ reported relief or absence of 
plantar heel pain in $52 \%$ of patients participating in exercises to stretch the plantar fascia, and $22 \%$ after stretching the Achilles tendon. Even though the pathophysiology of PF is different from that of FPA, Achilles tendon stretching can be suggested as a part of treatment. Studying the effect of exercise intervention to reduce risk and lower incidence would be worthwhile.

Relationship between heel spur and plantar heel pain has been controversial. Davies et al. ${ }^{16}$ and Williams et al. ${ }^{17}$ checked for heel spurs in more than $50 \%$ of patients with plantar heel pain. Dimarcangelo and $\mathrm{Yu}^{18}$ looked for it in $50 \%$ of patients with PF, and some asymptomatic subjects or subjects without PF. This study showed that $38.3 \%$ of patients with PF had spurs, compared with $13.5 \%$ of those with FPA $(p=0.001)$. No significant differences between $\mathrm{PF}$ and heel spur by multinomial logistic regression were observed.

A limitation of this study is that treatment and evaluation of effects after treatment were not investigated. For example, inferior heel pain from PF can be relieved with a prefabricated insole or heel cushion cup to minimize extension of the plantar fascia. Stretching exercises of the plantar fascia and Achilles tendon cab also relieved pain. An alternative treatment may be steroid local injection, or extracorporeal shock wave therapy for intractable pain. Heel cushions, heel cups, or low-dye taping can be applied to relieve the pressure on the calcaneus of FPA patients. ${ }^{1-3}$

Accurate diagnosis is important to evaluate the response of treatment and prognosis of disease because treatment such as local steroid injection can cause the atrophied tissue to shrink further in FPA patients. This study determined the causes of plantar heel pain in 250 subjects and gathered basic data on the incidence of the causes of plantar heel pain, and clinical differences and differential diagnosis in 133 subjects with PF and 37 patients with FPA. In the future, we also plan to evaluate the response to treatment.

\section{CONCLUSION}

Plantar heel pain can be caused by not only PF or FPA, but various other causes as well. Patients with PF or FPA typically show different characteristics of clinical features about timing of pain, tender site, and bilaterality. Plantar heel pain requires differential diagnosis with consider- ation for appropriate management.

\section{REFERENCES}

1. Buchbinder R. Clinical practice. Plantar fasciitis. N Engl J Med 2004; 350: 2159-2166

2. Bordelon RL. Subcalcaneal pain. A method of evaluation and plan for treatment. Clin Orthop 1983; 177: 49-53

3. Neufeld SK, Cerrato R. Plantar fasciitis: evaluation and treatment. J Am Acad Orthop Surg 2008; 16: 338346

4. Lemont $\mathrm{H}$, Ammirati KM, Usen N. Plantar fasciitis: a degenerative process (fasciosis) without inflammation. J Am Podiatr Med Assoc 2003; 93: 234-237

5. Riddle DL, Pulisic M, Pidcoe P, Johnson RE. Risk factors for plantar fasciitis: a matched case-control study. J Bone Joint Surg Am 2003; 85-A: 872-877

6. Baxter DE, Pfeffer GB. Treatment of chronic heel pain by surgical release of the first branch of the lateral plantar nerve. Clin Orthop Relat Res 1992; 279: 229236

7. Karabay N, Toros T, Hurel C. Ultrasonographic evaluation in plantar fasciitis. J Foot Ankle Surg 2007; 46: 442-446

8. Rhee WI, Kim JM. Ultrasonographic findings of thickness and compressibility of heel pads in outpatient. J Korean Soc Med Ultrasound 2004; 23: 213-218

9. Gould N. Graphing the adult foot and ankle. Foot Ankle 1982; 2: 213-219

10. McCarthy DJ, Gorecki GE. The anatomical basis of inferior calcaneal lesions. A cryomicrotomy study. J Am Podiatr Med Assoc 1979; 69: 527-536

11. Pfeffer G, Bacchetti P, Deland J, Lewis A, Anderson R, Davis W, Alvarez R, Brosdsky J, Cooper P, Frey C, et al. Comparison of custom and refabricated orthoses in the initial treatment of proximal plantar fasciitis. Foot Ankle Int 1999; 20: 214-221

12. Riddle DL, Schappert SM. Volume of ambulatory care visits and patterns of care for patients diagnosed with plantar fasciitis: a national study of medical doctors. Foot Ankle Int 2004; 25: 303-310

13. Forman WM, Green MA. The role of intrinsic musculature in the formation of inferior calcaneal exostoses. Clin Podiatr Med Surg 1990; 7 : 217-223

14. Inman VT, Ralston HJ, Todd F. Human walking, 2nd 
ed, Baltimore, Williams \& Wilkins, 1994, 45-72

15. Digiovanni BF, Nawoczenski DA, Malay DP, Graci PA, Williams TT, Wilding GE, Baumhauer JF. Plantar fascia-specific stretching exercise improves outcomes in patients with chronic plantar fasciitis. A prospective clinical trial with two-year follow-up. J Bone Joint Surg 2006; 88: 1775-1781

16. Davies MS, Weiss GA, Saxby TS. Plantar Fasciitis: how successful is surgical intervention? Foot Ankle Int 1999; 20: 803-807

17. Williams PL, Smibert JG, Cox R, Mitchell R, Klenerman L. Imaging study of the painful heel syndrome. Foot Ankle 1987; 7: 345-349

18. Dimarcangelo MT, Yu TC. Diagnostic imaging of heel pain and plantar fasciitis. Clin Podiatr Med Surg 1997; 14: 281-301 